\title{
El espacio social cubano y el sector económico mixto o privado ${ }^{1}$
}

Juana Elvira Suárez Conejero²

1 derrumbarse el campo socialista y dadas las presiones interna-
cionales, fundamentalmente el bloqueo de los Estados Unidos,
Cuba se encuentra condicionada a realizar algunos cambios en su macroestructura económica, política y social. El conjunto de medidas de subsistencia y desarrollo derivadas de la política de Período Especial en Tiempo de Paz constituyen la expresión objetiva de las transformaciones estructurales e inciden en la composición del espacio social cubano, donde, entre otras, lo económico empieza a potenciar efectos simbólicos junto a la alta y sostenida politización del sistema de relaciones sociales.

El movimiento de la fuerte estatalización hacia la flexibilización y los mecanismos de oferta demanda, la sustitución paulatina del igualitarismo y paternalismo estatal por necesarias políticas sociales, solidarias en sus principios, pero ni excesivamente igualitaristas ni paternalistas, han tenido consecuencias directas sobre los cubanos, y sobre sus representaciones del mundo social.

La introducción del sector económico mixto o privado a través de la inversión extranjera, como consecuencia de la reestruccturación interna, ha sido el cambio estructural más importante acaecido en la Cuba socialista. En consecuencia, los individuos ahora se desplazan, conscientemente o inconscientemente, o se someten a la inercia en el espacio de las relaciones sociales y estos movimientos no se realizan en el " vacío ", ni al

1 Un artículo similar de la autora fue publicado, en francés, en la Revista Alternatives Sud, editada por Centre Tricontinental de Louvain-la-Neuve, Bélgica y L'Harmattan de París, Francia.

2 La autora es Doctora en Sociología de la Universidad Católica de Lovaina, Bélgica. 
“ azar ", lo que nos hace suponer la posibilidad de una reestructuración de los diferentes grupos sociales. Reestructuración, que a su vez, resulta necesaria para realizar la inserción de Cuba en el sistema internacional de relaciones comerciales y políticas.

Sin embargo, resultaría muy difícil en un artículo, poder abarcar exhaustivamente nuestro tema de análisis. Es por ello que vamos a centrar la atención en algunos elementos de interés: las transformaciones estructurales, los agentes del cambio y las representaciones sociales, orientadas hacia el posicionamiento y re - posicionamiento dentro del sector económico ${ }^{3}$ no estatal, es decir, poder comprender las transformaciones que se producen en la economía privada o mixta en $\mathrm{Cuba}^{4}$ como consecuencia de la inversión extranjera, a nivel de estructura, agentes y representaciones.

\section{La Revolución cubana y la crisis económica de los 90}

Como es conocido, en 1959 triunfó en Cuba un movimiento de liberación nacional y 2 años después, en 1961, se declaró por parte de la dirección del país el carácter socialista del proceso revolucionario. Entre los rasgos básicos de la composición socio - económica de la sociedad cubana antes del triunfo, tenemos: el bajo peso de los obreros con escasa calificación y preparación profesional, el alto peso del campesinado pobre no poseedor de la tierra, la proporción relativamente alta de sectores medios, la exigua presencia de una intelectualidad y la ausencia casi total de imbricación entre el sistema educacional con las necesidades de formación de fuerza de trabajo calificada. Por otra parte predominaban sectores no interesados en una vía de desarrollo capitalista nacionalista, es decir, una burguesía comercial exportadora - importadora con intereses de comercio fundamentales respecto a los Estados Unidos, lo que implicó la inexistencia de una burguesía auténticamente nacional.

3 Que utilicemos la variable sector económico como primer intento de reducción no quiere decir que ignoremos la complejidad del fenómeno y lo reduzcamos al economismo, lo que queremos situar es un primer corte, resultado evidente de las transformaciones macro - estructurales.

4 Cuando hablamos de sector no estatal o mixto nos referimos concretamente a los de entidades mixtas con capital cubano y extranjero, y a las inversiones $100 \%$ extranjeras. 


\section{EL ESPACIO SOCIAL CUBANO Y EL SECTOR ECONÓMICO MIXTO O PRIVADO /}

Si examinamos las principales medidas llevadas a cabo en los diez años de la Revolución, vemos que se observó un fuerte y acelerado ritmo de liquidación de la antigua estructura imperante. Como dato de interés podemos decir que, en 1960, a solo un año del triunfo revolucionario, el Estado controlaba casi el 100\% de la industria y el comercio, y en 1963 controlaba el $70 \%$ de la producción agrícola ${ }^{5}$.

\section{Las principales medidas político - económicas fueron:}

- La nacionalización de grandes empresas industriales, comerciales, bancarias, etc., las cuales hicieron desaparecer las bases económicas capitalistas y formaron la base de la propiedad estatal o propiedad social de todo el pueblo.

- El desmantelamiento de la pequeña propiedad urbana.

- La primera y segunda Ley de Reforma Agraria, eliminándose de esta manera la gran propiedad privada sobre la tierra.

Como se puede apreciar, estas medidas condujeron a una fuerte estatalización, es decir, implicaron un fortalecimiento numérico de la propiedad estatal. Las ventajas de estas políticas son conocidas. Si comparamos Cuba antes de 1959 y en 1989 podemos apreciar las resultantes positivas $^{6}$ :

\begin{tabular}{|l|c|c|}
\hline & $\mathbf{1 9 5 8}$ & $\mathbf{1 9 8 9}$ \\
\hline $\begin{array}{l}\text { Mortalidad Infantil } \\
\text { (por mil nacidos vivos) }\end{array}$ & Más de 60 & 11.1 \\
\hline Esperanza de vida al nacer en años & 62.29 & 74.27 \\
\hline Graduados de educación primaria & 21.600 & 155.000 \\
\hline Cantidad de médicos & 3.960 & 34.752 \\
\hline $\begin{array}{l}\text { Tenencia de bienes de uso duradero por } \\
\text { mil habitantes (TV) }\end{array}$ & 46.6 & 173.0 \\
\hline Densidad telefónica (por mil habitantes) & 27.5 & 52.3 \\
\hline
\end{tabular}

5 Martínez, A., El plan de la economía nacional, Revista Cuba Socialista, número 31, La Habana, 1964.

6 Comité Estatal de Estadística, Cuba en Cifras, La Habana, 1990. 
Sin embargo, esto trajo consigo, entre otras consecuencias, un crecimiento excesivo y desproporcionado del grupo de los empleados administrativos, dirigentes y especialistas en actividades de planificación y control. A mediados de los años setenta se extendió la propiedad cooperativa agropecuaria y se conservó un sector relativamente escaso de pequeños agricultores privados y trabajadores urbanos por cuenta propia. El peso de este sector no estatal era muy débil y predominaban en la política socioeconómica las medidas destructivas de la pequeña propiedad. En síntesis, puede decirse que hasta los años 80 el modelo de organización de las relaciones de propiedad en Cuba identificó la propiedad socialista con un alto grado de estatalización, impidiendo el despliegue de otras formas de propiedad y sobrecargando al Estado con funciones económicas y administrativas y convirtiéndolo así, en el casi único responsable de proveer fuentes de empleo e ingresos estables a la gran mayoría de la población.

Un segundo rasgo de la sociedad cubana hasta 1989 fue las difusas fronteras, a nivel de representaciones, entre los principales grupos sociales, ya que el despliegue de políticas igualitaristas ${ }^{7}$, fundamentadas en la igualdad de oportunidades y en un sistema de gratuidades, contribuyó a la conformación de representaciones en los diferentes actores acerca de la no existencia de diferencias entre ellos.

Ejemplo de ello, podemos citar la masificación de la educación; su carácter gratuito y no selectivo posibilitó en la práctica, mayores oportunidades de movilidad social a los diferentes agentes. Sin embargo, esto trajo el predominio de los "trabajadores intelectuales", o al menos, personal con un elevado nivel educacional dentro de los sectores productivos o de servicios. Estas políticas a su vez, no sólo incidieron en la esfera de las representaciones sociales sino que trajeron consecuencias tangibles, como la relativa pérdida de valor simbólico en los títulos de enseñanza, ya que, la cantidad de individuos con títulos universitarios o técnicos resultó muy por encima de las necesidades sociales, dada la masificación de la educación.

7 Podemos contar, entre estas políticas, la igualdad de oportunidades y gratuidad de la educación, salud pública, deportes, manifestaciones artísticas, etc. 


\section{EL ESPACIO SOCIAL CUBANO Y EL SECTOR ECONÓMICO MIXTO O PRIVADO I}

Una tercera característica de la sociedad cubana antes de 1989 fue la alta

politización del sistema de relaciones sociales, sostenido por el apoyo masivo, y que conllevó a que lo político tuviera importantes efectos en detrimento de lo económico. Para comprender esta idea es necesario señalar que Cuba se enfrenta, al triunfar la Revolución, a las amenazas directas de los Estados Unidos, materializadas en el bloqueo económico, agresiones militares, ideológicas, bacteriológicas, lo que conlleva a que sea el bloque socialista y fundamentalmente la URSS, quienes brinden el apoyo económico y político necesario a la Revolución Cubana. Por otra parte, el carácter popular del proceso revolucionario, reforzado por una tradición histórica de siglo y medio de luchas contra la dominación extranjera (primero contra España durante el siglo XIX y luego contra los Estados Unidos en todo el transcurso del siglo XX) implicaba un gran apoyo al proceso de la mayor parte de los grupos sociales. Todo ello, junto al prestigio obtenido en las luchas pre y posrevolucionarias y la personalidad de su líder Fidel Castro, hicieron que lo político pasara a tener importantes efectos.

En resumen, comprender el espacio de las relaciones sociales cubano antes de 1989 implica considerar la importancia del factor político. El capital económico, por su parte, resultó altamente desvalorizado por los agentes y a través de sus representaciones. Por una parte, su tenencia era incongruente con los principios del sistema socialista, contrarios al modelo que imperaba antes de la Revolución. Por otra parte, al estar Cuba insertada en el sistema de comercio socialista CAME, lo económico daba paso a la valorización de otras representaciones, en el sentido de la solidaridad entre países socialistas, la defensa del sistema político, etc., es decir, lo político se potenció a nivel de las representaciones sociales en detrimento de lo económico.

Una cuarta característica del socialismo cubano era que los agentes conformaron sus representaciones y en consecuencia actuaban en favor de un ideal social, dejando en un segundo plano las individualidades; el ideal comunista, el cual considera como lo más importante al hombre comunista o "hombre nuevo", cuya máxima expresión es el Che, es la expresión del sacrificio individual a favor de la sociedad. Esta representación resultaba y resulta compartida por la mayor parte de los cubanos dadas las respuestas histórico concretas que la Revolución ha brindado y brinda. 
Las condiciones socio económicas concretas conllevaron, evidentemente, a que existiera este sistema de sentido y no otro. El hombre nuevo y la liberación social son el soporte fundamental de la existencia misma de la Revolución cubana y las anteriores luchas por la toma del poder contra la injerencia extranjera, siendo también consecuencia de las difíciles condiciones que vivía y vive el país frente a la hostilidad de los Estados Unidos. Todo esto condujo a que en la sociedad cubana existiera la potenciación de lo social por encima de lo individual.

En resumen, para poder comprender la sociedad cubana hasta 1989 es necesario reconocerla como una sociedad donde:

1. Las formas de propiedad y de retribución de la riqueza eran esencialmente estatales.

2. Las fronteras entre los grupos sociales se hicieron difusas y por tanto las representaciones acerca de ellos, como consecuencia de la fuerte igualitarización.

3. Lo político alcanzaba importantes efectos en detrimento de lo económico.

\section{Se potenciaba lo social por encima de lo individual}

A partir del derrumbe del socialismo en Europa del Este y, específicamente, la desaparición de la URSS, Cuba se enfrenta a una grave crisis, fundamentalmente de tipo económico, acentuada por el recrudecimiento del bloqueo que mantienen los Estados Unidos a la Isla y por los errores que habían sido cometidos en la conducción de la economía socialista cubana.

En primer lugar, a lo largo de más de 30 años, Cuba había desarrollado crecientes relaciones con la URSS y los demás países de Europa del Este, al ser, como habíamos dicho anteriormente, uno de los países insertados en el sistema de cooperación y colaboración económica llamado CAME. Según fuentes oficiales cubanas $^{8}$, puede afirmarse que el $85 \%$ del comer-

8 Lage, Carlos, Conferencia sobre la situación económica cubana, 1er Encuentro La Nación y la Emigración, Revista Contrapunto, Año 5, Número 6, Edición 42, Junio de 1994, páginas 68 - 80. 


\section{EL ESPACIO SOCIAL CUBANO Y EL SECTOR ECONÓMICO MIXTO O PRIVADO /}

cio de la Isla se desarrolló con esos países. La tecnología y la industria que se importó e instaló durante todos estos años, eran esencialmente de la URSS y Europa del Este y la formación de profesionales cubanos en el exterior junto a la asimilación de tecnologías, estuvieron también, en buena parte, asociadas a estas relaciones.

A partir de 1989 se interrumpió esta cooperación. Ello trajo como consecuencia, en primer lugar, la pérdida de precios favorables, mutuamente ventajosos, para los productos cubanos de exportación, fundamentalmente el azúcar, principal renglón de la economía del país en aquellos momentos. Por otra parte, se paralizó aproximadamente el $70 \%$ de la industria nacional, se perdieron los principales mercados, se cancelaron posibilidades de créditos, de fuentes de financiamiento externas, para el desarrollo y el desbalance comercial con estos países, entre otras.

Según datos oficiales cubanos ${ }^{9}$, obsérvese el comportamiento del PIB de la isla en los primeros años de la crisis económica:

\begin{tabular}{|ll|}
\hline 1989 & +0.7 \\
1990 & -2.9 \\
1991 & -10.7 \\
1992 & -11.61 \\
1993 & -14.9 \\
\hline
\end{tabular}

Es decir, la economía cubana tuvo una caída de casi un 40\% entre 1990 y 1993. En resumen, se perdieron las relaciones de intercambio económico que se habían establecido a lo largo de 30 años con la URSS y Europa del Este y que atenuaban, hasta cierto punto, el bloqueo económico que los Estados Unidos mantenían hacia Cuba desde los inicios del triunfo revolucionario.

En la medida en que comienzan a presentarse estas dificultades, el bloqueo $^{10}$ hace sentir más sus efectos. La prohibición de acceder al mercado de los Estados Unidos, uno de los más importantes en el mundo y especialmente para América Latina, dada su cercanía geográfica, el considerable excedente de productos a precios inferiores en comparación con otros mercados, así como su fuerte desarrollo tecnológico, resulta,

9 Fidel Vascós González, Artículo del Periódico Granma (19/8/95), Oficina Nacional de Estadística, La Habana, Cuba, 1995.

10 Este constituye un conjunto de acciones económicas, comerciales, financieras, políticas y militares contra el país, que han ido acrecentándose en el tiempo, con leyes como la Torricelli y actualmente la Helms Burton. 
evidentemente, una manera de colocar a Cuba en una posición desventajosa para su economía, frente a los restantes países del área, y la obliga a buscar sus productos en mercados distantes. Asimismo, determinadas acciones políticas generan presiones en el mercado internacional sobre las condiciones en que Cuba comercia, lo que eleva los costos ante los riesgos que corren terceros en su comercio con la isla. Las presiones de Estados Unidos no permiten que el país tenga acceso al Fondo Monetario Internacional y al Banco Mundial, ni tampoco a otras fuentes de financiamiento, por lo que el desarrollo no puede contar con fuentes externas importantes.

Otra consecuencia del bloqueo, es la prohibición a ciudadanos norteamericanos de viajar a Cuba, lo que incide directamente en el desarrollo del turismo, otra fuente de ingresos importante para el país. El flujo de turistas se encuentra fundamentalmente en los países desarrollados, por lo que el mercado cubano tiene que recurrir principalmente a Europa, lo que acrecienta los costos al visitante y reduce su número. Por otra parte, el bloqueo incluye en su política presionar a empresarios extranjeros que comercian con los Estados Unidos a suspender sus actividades comerciales con Cuba.

Estudios realizados dan cuenta de que a lo largo de existencia del bloqueo Cuba ha perdido más de 60.000 millones de dólares estadounidenses a causa de sus efectos. Otras investigaciones plantean que sin los efectos del bloqueo económico, Cuba hubiera ingresado, por ejemplo, en el año 1994, un 40\% más en moneda libremente convertible de lo que ingresó, con las mismas producciones y los mismos precios ${ }^{11}$. Todo este panorama se complejiza aún más con la entrada en vigor de la Ley Torricelli y posteriormente con la controvertida Ley Helms Burton, la cual ha sido fruto de numerosas protestas internacionales por su carácter extraterritorial.

Finalmente, los errores propios del sistema socialista se hicieron sentir también con más fuerza en este momento. Ya desde 1984 había comenzado a gestarse, por parte de la dirección del país, el llamado "Proceso de rectificación de errores y tendencias negativas". Para comprender este 


\section{EL ESPACIO SOCIAL CUBANO Y EL SECTOR ECONÓMICO MIXTO O PRIVADO /}

proceso es necesario situarlo en su continuidad histórica. Según afirma Darío L. Machado, en su libro "Nuestro propio camino" se pueden concebir tres momentos fundamentales, o tres períodos desde el triunfo de la Revolución hasta la comúnmente llamada rectificación: el primer proyecto de desarrollo integral, la institucionalización y el proceso de rectificación ${ }^{12}$. Nos detendremos en esta última etapa, lo que no quiere decir que se pueda hacer un análisis de ella de manera aislada, ya que sus bases se crean en los momentos anteriores.

\section{Los principales errores detectados, entre otros, fueron:}

- Los resultados económicos internos se lograban con altos niveles de importación y con gastos de energía y medios de producción que no se correspondían con la eficiencia necesaria.

- Escaso dinamismo en el desarrollo de los renglones exportables; las inversiones no estaban dirigidas a la sustitución de importaciones.

- Existencia de mecanismos mercantiles de oferta y demanda en la distribución de los productos agropecuarios en el Mercado Libre Campesino.

- Ineficiencia de los mecanismos de planificación y dirección de la economía.

- No aprovechamiento a plenitud de las capacidades industriales y agrícolas estatales.

- Abandono de la construcción de obras sociales y viviendas, acumulándose recursos en inversiones que no debían hacerse o que no se terminaban.

12 Machado Rodríguez, Darío L., Nuestro propio camino, Análisis del proceso de rectificación en Cuba, Editora Política, La Habana, Cuba, 1993. 
- Inadecuación del trabajo científico-técnico a la economía.

- Existencia de privilegios, acomodamiento e irresponsabilidad de funcionarios.

- Inexistencia de mecanismos adecuados de estimulación, tanto morales como materiales que se correspondieran con los resulta dos alcanzados en la producción y servicios.

A mediados de 1986 se lanza la llamada "contraofensiva estratégica", como línea política a largo plazo para la búsqueda de soluciones a estos problemas. La primera medida implementada fue la supresión de las relaciones mercantiles basadas en la oferta y la demanda, en la comercialización de la producción agropecuaria del Mercado Libre Campesino. Más tarde, se adoptaron decisiones acerca del perfeccionamiento de los principales componentes del sistema de dirección de la economía, se hizo una reforma de los precios mayoristas y se emitieron normas para el funcionamiento de las uniones y empresas estatales.

No obstante todo este esfuerzo, este proceso no había culminado con todo el éxito requerido para Cuba en 1989. A pesar de que el sentimiento hacia el proceso revolucionario por parte de la población cubana era en general de confianza, el derrumbe del socialismo hizo que decayera la credibilidad en el bloque o sistema. Es por ello que, la dirección del país puso en un primer plano la argumentación de las posibilidades reales de mantenimiento del socialismo enfatizando en conceptos más concretos: exigencia, control, participación, reafirmación de la propiedad social, papel del Partido en la sociedad, necesidad de atención al hombre, simplificación y fortalecimiento de las estructuras empresariales, etc.

Como se puede apreciar, estas políticas no implicaban cambios estructurales en el país, sino que iban orientadas al perfeccionamiento del sistema sin afectar los cuatro principios esenciales que describimos anteriormente: continuaba la fuerte estatalización de los medios de producción, el igualitarismo o difusión representativa de las fronteras de los grupos sociales, la potenciación de lo social por encima de lo individual y, a pesar del derrumbe del socialismo en Europa del Este, los efectos de lo político por encima de lo económico conti- 


\section{EL ESPACIO SOCIAL CUBANO Y EL SECTOR ECONÓMICO MIXTO O PRIVADO /}

nuaban con gran fuerza, dado el unipartidismo, la autenticidad del movimiento de liberación nacional y el prestigio y autoridad moral de Fidel Castro, todo ello sumado a las ventajas que con evidencia había obtenido la población cubana con las políticas de la Revolución. Las transforaciones estructurales. Un conjunto de medidas para la reanimación económica.

Sin intentar hacer comprender 1989 como un punto exacto de ruptura (hemos tratado de ver la continuidad histórica del proceso), no es menos cierto que la conjunción de los tres factores: derrumbe del socialismo, bloqueo de los Estados Unidos y deficiencias del sistema econónico socialista cubano, llevan a efectuar, a partir de esta fecha, un relativo viraje en las políticas hasta ese momento establecidas.

El « Período Especial en tiempo de Paz $»^{13}$, que se enfrenta a partir de 1990 y que se mantiene hasta este momento, se puede comprender como la búsqueda de nuevas políticas de subsistencia y desarrollo ante la aguda crisis a la que se enfrenta el país.

Como podremos apreciar en el inventario de medidas tomadas a partir de ese año, los cambios en las estructuras comienzan a evidenciarse paulatinamente a partir de ese momento. El programa de reformas y transformación económica se inscribe dentro de una estrategia cuyo objetivo fundamental es la defensa de los intereses nacionales, Consolidar el socialismo y la Revolución Cubana. Las reformas realizadas comienzan a introducir cambios sustanciales en la estructura de la propiedad y la gestión sobre ésta.

La ley de inversiones (se permiten inversiones de capital extranjero de dos formas : en combinación con capital cubano para la gestión ya que la propiedad es estatal, o puramente extranjero) y la expansión del trabajo por cuenta propia ( los privados nacionales pueden implantar pequeños negocios, fundamentalmente de servicios, contribuyendo al Estado a través de una política de impuestos) aumentan el espacio para diferentes formas de propiedad no estatal. Por otra parte, los cambios

13 Nombre según se caracteriza la etapa de crisis que vive el país. 
en el sector agrícola (entrega de parcelas en usufructo, aumento de la cooperativización de la tierra del estado) hacen que la gestión sobre la propiedad sufra cambios significativos. En resumen, la tendencia en el país es la del crecimiento de la participación de sectores no estatales en su estructura. ${ }^{14}$ En consonancia con ello, el conjunto de medidas se encuentra encaminado a reforzar la descentralización y a permitir un mayor papel de las relaciones monetarias mercantiles, es decir, permitirle al mercado actuar bajo las leyes de la oferta y la demanda. Nótese en la siguiente tabla , la estimación de los efectos del programa de reformas sobre el funcionamiento de la economía. ${ }^{15}$

\begin{tabular}{|l|c|c|c|}
\hline Programa de medidas & $\begin{array}{c}\text { Tendencia a la } \\
\text { centralización }\end{array}$ & $\begin{array}{c}\text { Tendencia a la } \\
\text { descentralización }\end{array}$ & $\begin{array}{c}\text { Acción sobre el } \\
\text { mercado de } \\
\text { libre } \\
\text { oferta/demanda }\end{array}$ \\
\hline Apertura al capital extranjero & - & + & + \\
Desarrollo acelerado del turismo & - & + & + \\
Descentralización del comercio exterior & - & + & + \\
Despenalización del dólar 16 & - & + & + \\
Apertura de casas de cambio 17 & - & + & + \\
Cambios en el sector agrícola & - & + & + \\
Reactivación del trabajo por cuenta propia & - & + & + \\
Apertura del mercado agropecuario 18 & - & + & + \\
Ley de inversiones mixtas y $100 \%$ extranjeras & - & + & + \\
Inicio de la reforma bancaria ${ }^{19}$ & - & + & + \\
Ley tributaria ${ }^{20}$ & - & + & + \\
\hline
\end{tabular}

14 Triana Cordoví, Juan, EI desempeño de la economía cubana en 1995 y el proceso de transformaciones económicas,Revista Economía y Desarrollo, No. 1, 1996, Facultad de Economía, Universidad de La Habana, página 21.

15 Triana Cordoví, Juan, El desempeño de la economía cubana en 1995, Op. Cit. Página 22.

16 En Cuba, hasta 1993, la tenencia de dólares estadounidenses o cualquier otra moneda diferente a la del país, era penalizada judicialmente.

17 Las casas de cambio en divisas permiten cambiar el peso cubano "corriente" en el peso cubano convertible (equivalente 1 peso convertible a 1 al dólar estadounidense) y viceversa.

18 Esta medida posibilita a los productores agrícolas vender los excedentes de su producción a precios libres bajo un sistema tributario de pago de impuestos al Estado.

19 Descentraliza las operaciones de la banca cubana y revitaliza el uso de instrumentos comunes a esta esfera.

20 Flexibiliza la ejecución del presupuesto por parte de las empresas y regula los impuestos a pagar por los propietarios nacionales y extranjeros. 


\section{EL ESPACIO SOCIAL CUBANO Y EL SECTOR ECONÓMICO MIXTO O PRIVADO /}

En resumen, la primera característica que sustentaba el desarrollo económico cubano, el sistema de relaciones sociales y las representaciones en consecuencia, antes de 1989, es decir, la fuerte estatalización en la producción de bienes y distribución de la riqueza, comienza a presentar un relativo viraje hacia la flexibilización, descentralización y consecuentemente, hacia el mercado de libre oferta demanda, como resultado del conjunto de medidas tomadas en el Período Especial.

Por otra parte, según estimaciones oficiales cubanas, está creciendo el número de empleados en el sector no estatal (alrededor de un $20 \%$ de la fuerza de trabajo) lo que refuerza la tendencia al cambio de estructura del empleo por formas de propiedad. No obstante, se presenta una contradicción entre la calidad de la oferta de empleo y las aspiraciones individuales (recuérdese el alto nivel educacional alcanzado por gran parte de la población cubana), lo que se manifiesta en una tendencia en el movimiento del empleo hacia sectores de alta remuneración pero de menos calificación. Ello es resultante de un proceso de diferenciación social, públicamente reconocido, como consecuencia de las transformaciones estructurales.

Para comprender bien esta idea, es necesario saber que, al despenalizarse en 1993 el dólar estadounidense en Cuba, comienza a potenciarse paralelamente un proceso de apertura del mercado de bienes de consumo y servicios en esta moneda. La crisis económica tiene una expresión en la mayor parte de la población cubana, fundamentalmente en cuanto a las posibilidades de adquisición de productos esenciales para la subsistencia. ${ }^{21}$ A partir de ese momento, una de las maneras más factibles de adquisición de dichos productos necesarios es la utilización de este mercado en moneda libremente convertible, sin embargo, prácticamente ningún empleo en la isla es remunerado en divisas. El canje del peso cubano respecto al dólar estadounidense era, en 1993, en el mercado negro, 120 pesos por un dólar, y recientemente, ya de manera oficial, resulta cercano a los 21 pesos por un dólar estadounidense. Este indicador muestra la mejoría paulatina de la economía cubana, pero de todas formas la situación económica individual sigue siendo muy difícil para la mayor

21 No obstante, el Estado cubano garantiza un conjunto de productos subsidiados por igual para toda la población. 
parte de los cubanos. Por otra parte, los precios del mercado en moneda libremente convertible son altos, producto de la política expresada en que lo elevado de los precios permite hacer redundar los beneficios en toda la población, sobre todo incluyendo a los que se encuentran fuera de esta economía, a través de políticas sociales como principio de la redistribución más justa de la riqueza.

Esto potenció un conjunto de nuevas estrategias individuales y colectivas para la obtención de los productos esenciales, entre otras a través de la tenencia del dólar estadounidense. Decimos que las estrategias son individuales y colectivas, porque consideramos la "marca particular individual" como desviación de regularidades colectivas condicionadas según los diferentes grupos sociales.

\section{Entre las estrategias más recurridas podemos señalar:}

- Búsqueda de una entrada adicional de dinero cubano, dólar estadounidense o productos, como resultado de una doble contratación a nivel estatal o privado.

- Búsqueda de un empleo altamente remunerado de manera legal, en dinero o productos, como resultado de la contratación en el sector mixto o privado.

- Búsqueda de entrada adicional de dólar estadounidense o productos por medio de contratos de trabajo temporales fuera del país.

- Búsqueda de entrada adicional de dólar estadounidense o productos a través de la familia o amigos residentes en el exterior.

- Búsqueda de entrada adicional de dinero cubano, dólar estadounidense o productos, por vías no legales (inserción en el mercado negro, robo, proxenetismo, etc.)

- Búsqueda de entrada adicional de dinero cubano, dólar estadounidense o productos, por vías legales pero sancionadas por el 


\section{EL ESPACIO SOCIAL CUBANO Y EL SECTOR ECONÓMICO MIXTO O PRIVADO /}

imaginario colectivo (prostitución).

Nótese que las tres primeras estrategias se vinculan al trabajo considerado en el marco jurídico legal, no así las restantes. Sin embargo, esto no quiere decir que los empleos se puedan por el momento remunerar en dependencia del aporte social del trabajo. Es decir, la obtención de ventajas económicas, incluso en los casos en que es producto del trabajo, no se corresponde en ocasiones con el aporte, sino con el tipo de empleador, la forma de remuneración que utiliza o el tipo de trabajo. Por ejemplo, los trabajadores del sector turístico de baja especialización (camareros, dependientes, etc.), tienen más probabilidades de obtener dólares en el sentido de que la cercanía que tienen con esta moneda les proporciona mecanismos relativamente estables para su obtención, como son la propina, los regalos de los turistas, etc. Por otra parte, quienes trabajan en firmas extranjeras, a pesar de que la remuneración la realiza el Estado cubano en pesos, cuentan con posibilidades de acceso a la "economía del dólar" al recibir en muchos casos dinero en efectivo o ventajas en productos por parte del empleador o los clientes. Esto no sucede así en una parte de los empleados estatales, que aunque su trabajo pueda aportar más a la sociedad, o sea, más calificado, no tienen, generalmente acceso a la divisa como resultado de su aporte y en consecuencia, su empleo no les permite entrar a este mercado. Los privados nacionales logran entradas considerables en dinero cubano o en divisa a partir del establecimiento de precios muy elevados a sus productos o servicios respecto al salario medio en el país.

Los condicionamientos estructurales y, por tanto, las estrategias que se desarrollan, conllevan a la manifestación de diferencias entre los agentes y grupos sociales, con respecto a la tenencia de capital económico, en volumen y estructura, lo que no quiere decir que esta sea la única variable productora de diferencias sociales, sino que la manifestación de ellas se aprecia fácilmente en lo económico, contrariamente a lo que sucedía antes de 1989. En resumen, el Estado cubano deja de ser, como consecuencia del programa de medidas, la sola fuente generadora de vías para la satisfacción de las necesidades, teniendo que asumir los propios agentes, una parte de la resolución de sus necesidades básicas. 
Todo ello se inscribe en la actualidad, donde se muestra que lo social está dejando un espacio necesario a lo individual, sin que lo individual sea lo que prime. Las conquistas sociales de la Revolución se han mantenido (educación y salud pública gratuitas, deporte masivo, etc.) a pesar de la crisis económica y continúan centralizadas por el Estado para de esta manera poder garantizarlas, lo que ha conllevado a que determinados sectores se encuentren excluidos de hecho de la entrada en la economía mercantil privada o mixta, con todo un cuerpo jurídico que lo justifica en algunos casos (médicos, maestros, etc.) y que a su vez garantiza el mantenimiento de las conquistas revolucionarias. Sin embargo, esta exclusión jurídica no es garante de que los agentes implicados no acudan a algunas de las estrategias anteriormente descritas. En resumen, lo individual se encuentra contaminando lo social, sin que lo individual sea necesariamente lo que prime.

Desde otro ángulo los principios ideológicos de la Revolución, compartidos por gran parte de la población cubana siguen siendo los mismos. A pesar de que las condiciones internas y externas han cambiado para Cuba, la mayor parte de la población considera necesario mantener el poder revolucionario ${ }^{22}$, máxime ante el debilitamiento de las ideas comunistas después del derrumbe del socialismo en Europa del Este y por otra parte dada la cercanía y el diferendo entre Cuba y los Estados Unidos. Ante la urgencia del país de readaptarse a las nuevas condiciones internacionales y nacionales, se requiere encontrar un equilibrio entre los principios ideológicos que lo sustentan, el mundo capitalista exterior en el cual hay que insertarse y las consecuentes transformaciones y crisis económica interna. La propia complejidad de este equilibrio conlleva a que, en los diferentes agentes, se produzcan diferentes efectos al nivel de sus representaciones y sus prácticas sociales.

Otro elemento a tener en cuenta es que durante todo el proceso revolucionario anterior a 1989, el argumento del sacrificio a favor de la sociedad y futuras generaciones, funcionaba como resorte compulsor sico - afec-

22 En 1992, en lo que podemos calificar uno de los momentos más difíciles que atravesó el país, se realizaron elecciones directas y secretas, cuyo escrutinio fue público, y la dirección del país obtuvo un apoyo a través del voto unido de la mayoría de los cubanos. Los procesos eleccionarios posteriores han tenido un similar comportamiento. 


\section{EL ESPACIO SOCIAL CUBANO Y EL SECTOR ECONÓMICO MIXTO O PRIVADO /}

tivo, ya que a pesar de que la resultante era intangible, pues quedaba en el futuro, no era menos cierto la existencia de un importante grado de confianza en que ello funcionaría, junto a los logros que objetivamente se alcanzaban. Al comenzar la crisis, el poder revolucionario reafirma la vía socialista, pero expresa que la salida de los problemas dentro de ella sería a muy largo plazo, lo que implica que determinados agentes comiencen a cuestionar el sacrificio realizado y por hacer, aunque, como lo confirman numerosas investigaciones, en Cuba la mayor parte de las posiciones se adhieren a los principios de la Revolución. En Cuba existe un fuerte sentimiento antimperialista, condicionado por todas las luchas contra ese país durante el transcurso del siglo XX y para la mayor parte de los cubanos, el desarrollo del país sólo será posible con el éxito de la política de subsistencia y desarrollo, concebida desde dentro de la propia Revolución y que preserve los logros sociales.

Otro lado del análisis, debe tener en cuenta la política de desestabilización o subversión que los Estados Unidos mantienen hacia la isla, a través de agresiones radiales, televisivas y hasta biológicas. Si sumamos a esto las características de Cuba antes de 1989 y que los cambios estructurales actuales implican la entrada de lo exterior hacia la isla, podemos comprender que lo económico comience a producir efectos en las representaciones del mundo social en algunos agentes. Súmese también el relativo éxito de la economía de oferta y demanda como salida a la crisis cubana y las ineficiencias que aún subsisten en determinados mecanismos socialistas de administración y gestión económica. Es decir, lo económico comienza surtir efectos a nivel de las representaciones, frente a la sostenida y alta politización del sistema de relaciones sociales cubano.

En resumen, las características analizadas de la estructura del espacio social cubano y las representaciones que existían dentro de la población acerca de este antes de 1989, comienzan a modificarse con la introducción de los cambios estructurales, como se puede apreciar en el cuadro 


\section{Hasta 1989}

Fuerte control estatal sobre la producción de bienes materiales y distribución de la riqueza

\section{A partir de 1989}

Tendencia hacia el crecimiento de la participación de sectores no estatales en la producción, gestión y distribución de la riqueza.

Se hacen difusas las fronteras entre los grupos y, por tanto, de las representaciones acerca de ellos, como consecuencia de la fuerte igualitarisación social.

Importantes efectos de lo político sobre lo económico, en la conformación de representaciones.

Potenciación de lo social en detrimento de lo individual.
Inicio de un proceso de diferenciación social, que conlleva a manifestaciones expresadas fundamentalmente en el orden económico.

Lo económico comienza a surtir efectos en las representaciones, frente a la sostenida y alta politización del sistema de

Lo individual se encuentra contaminando lo social. relaciones sociales cubano.

que mostramos a continuación:

Todo ello nos conduce a suponer que la estructura de división en clases de la sociedad cubana ha sufrido transformaciones en consecuencia. Resulta evidente primero, que las transformaciones respecto a la propiedad y distribución de la riqueza han provocado movimientos de los agentes en el espacio de las relaciones sociales, trayendo como consecuencia, entre otras, un proceso de enmarcación de las anteriormente difusas fronteras entre los grupos, tanto en el sentido de las clases objetivadas, como de las incorporadas a través de las representaciones. La potenciación de lo individual y su contaminación de lo social, así como los nuevos efectos que potencia el capital económico, son también elementos a tener en cuenta para comprender las posibles transformaciones que se puedan estar dando en la estructura clasista cubana.

Sin intentar adherirnos a una posición estructuralista o puramente de determinismo económico y sin ignorar la complejidad teórica del tema, 


\section{EL ESPACIO SOCIAL CUBANO Y EL SECTOR ECONÓMICO MIXTO O PRIVADO /}

no es menos cierto que en una primera aproximación tenemos que ver los cambios estructurales en una doble dialéctica con respecto a las clases o grupos que se conforman en el espacio social cubano, es decir, hay que comprender los cambios clasistas como consecuencia de las transformaciones estructurales y por otra parte, es necesario integrarle a ello el activo papel que juegan los agentes en la conformación del sistema de relaciones sociales, sus transformaciones y las representaciones que se conforman acerca de él.

\section{Los agentes de la transformación. ¿Se crea una nueva clase social en Cuba?}

Si partimos del punto de vista marxista clásico para poder comprender si las transformaciones estructurales acaecidas en Cuba (nos referimos específicamente a la introducción del sector económico mixto o privado a través de la inversión extranjera) están llevando a conformar un nuevo grupo social, debemos, en primer lugar, adherirnos a la definición leninista de las clases sociales, en el entendido de conceptualización de los postulados teóricos de Marx.

Lenin plantea : "Las clases sociales son grupos de hombres que se distinguen por el lugar que ocupan en un sistema históricamente determinado, por su relación (la mayor parte del tiempo fijada y consagrada por las leyes) con los medios de producción, por el rol en la organización social del trabajo y por los medios de obtención y la importancia de la parte de las riquezas sociales de que disponen ${ }^{\prime 23}$.

Comencemos por un primer elemento a tener en cuenta en nuestro análisis y que será entonces el de la relación de los cubanos con respecto a la propiedad de los medios de producción en este sector. Para comprender este punto es necesario señalar que la Ley de Inversiones en Cuba se restringe a los extranjeros, es decir, los cubanos nacionales no pueden realizar inversiones personales o de provecho personal. Los representantes nacionales del sector (los llamaremos dirigentes empresarios) son

23 Lenin, V. Ilich, La gran Iniciativa, 1919, Obras Completas, Moscu, Editorial Progreso, tomo 6, página 425 . 
asalariados estatales, representan los intereses del Estado y las ganancias van enteramente a este último. Por lo tanto, el tema de la propiedad en este sector reduce a dos tipos de propietarios : los extranjeros que realizan la inversión y el Estado cubano. Los cubanos que habitan la isla están excluidos jurídicamente del tema de la propiedad privada a gran escala.

Un segundo elemento a tener en cuenta es que el estudio y revisión del cuerpo jurídico alrededor del sector mixto o privado en Cuba, muestra, en primera instancia, la imposibilidad de la acumulación de capital económico en personas naturales nacionales por vías diferentes a las de la corrupción.

Para tener una clara idea de cómo está constituído este cuerpo jurídico y de su rigor, vale solo señalar que la entrada al sector es obligatoria a través de agencias empleadoras del Estado cubano, quienes se encargan de los procesos de verificación de personal así como del ajuste salarial. En resumen, no existen vías legales para que los trabajadores cubanos del sector reciban dólares por concepto salarial o cantidades de pesos desproporcionadamente altas con respecto a la media nacional de salarios.

En segundo lugar, la pirámide salarial y de estimulación material resulta invertida. Un ejemplo simple, un empleado de un hotel al servicio del turismo internacional, recibe generalmente una propina en dólares (bajo diversos criterios) que hacen que su salario real sea mucho mayor que el que reciben en pesos. Sin embargo, el presidente de una compañía hotelera, no tiene derecho a esta estimulación generalmente. Ello ha traído como consecuencia que el flujo de movilidad se realice descendiendo en la escala de calificación laboral, pero a su vez es garante de la confiabilidad de los dirigentes del sector. Recordemos que es este grupo precisamente, el de los dirigentes empresariales, el que puede tener más posibilidades de acumulación importante de capital económico y por tanto constituirse como clase.

Por otra parte, el grupo de dirigentes empresariales está rodeado de un control estatal que, según las opiniones que expresan en un conjunto de

24 Las entrevistas fueron realizadas a una muestra significativa de empresarios dirigentes del sector en Ciudad de La Habana, en el marco de una tesis doctoral auspiciada por el Centro de Estudios sobre América, en Cuba, y la Universidad Católica de Lovaina en Bélgica. 


\section{EL ESPACIO SOCIAL CUBANO Y EL SECTOR ECONÓMICO MIXTO O PRIVADO /}

entrevistas realizadas a una muestra de ellos ${ }^{24}$, consideran excesivos. El número de auditorías, controles gubernamentales, etc. resulta también una garantía para evitar acumulaciones ilegales de capital económico.

La selección del personal tampoco se realiza arbitrariamente. Los dirigentes del sector son nombrados en general por la dirección del país y en su mayoría provienen de instituciones políticas. Dirigentes de la Juventud comunista cubana, jubilados de los cuerpos armados, etc. son quienes componen con mayor frecuencia la categoría dirigente del sector. La mayoría también son militantes del Partido Comunista, y por lo tanto, enfrentan una doble exigencia en cuanto al cumplimiento de sus funciones, exigencia política y exigencia administrativa. Vale añadir a esto que todo dirigente del sector debe firmar un Código de Ética, como elemento jurídico de compulsión a representar los intereses de la sociedad y del Estado cubano. En resumen, la entrada y permanencia en el sector no se ejecuta al azar, sino a través de mecanismos que garantizan la selección de dirigentes empresariales acordes con los principios de la Revolución cubana, y acordes a la política actual de subsistencia y desarrollo desde una perspectiva socialista, y que obligan a minimizar las tentativas de corrupción a través del control estatal.

Es decir, tomando la definición leninista de clases sociales, podemos concluir que en Cuba no existen las condiciones objetivas para la conformación de una nueva clase social, ni siquiera desde la perspectiva de "clase en sí ” señalada por Marx, ya que :

- Los cubanos que habitan la isla están excluidos jurídicamente del tema de la propiedad privada a gran escala

- Resulta imposible la acumulación de capital económico en personas naturales nacionales por vías diferentes a las de la corrupción.

- La entrada y permanencia en el sector no se ejecuta al azar, sino a través de mecanismos que garantizan la selección de dirigentes empresariales acordes con los principios de la Revolución cubana y acordes a la política actual de subsistencia y desarrollo desde una perspectiva socialista y que obligan a minimizar las tentativas de corrupción a través del control estatal. 
Sin embargo, consideremos el tema de las clases sociales desde otra perspectiva teórica. Por circunstancias históricas concretas la sociología socialista no estudió suficientemente el lado subjetivo, sicológico e individual, es decir, no estudió el activo papel de los agentes en la conformación de las clases sociales ni tampoco abordó críticamente las lógicas diferencias que se dibujaban en el espacio social. Este déficit, a nuestro juicio, lastró el desarrollo de la teoría marxista y se produjo a pesar de que, los propios clásicos del marxismo, planteaban lo contrario, como puede apreciarse en el ensayo de F. Engels: " La situación de la clase obrera en Inglaterra"

En resumen, la mayor parte de los estudios realizados desde el socialismo sobre las clases sociales en el socialismo desconocen una estructura clasista (el Estado, como entidad abstracta es el detentor de los medios de producción, por lo tanto, los actores se sitúan al mismo nivel en cuanto al problema de la propiedad) mientras que otros estudios abordan clasificaciones oficiales considerándolas grupos o clases (obreros, dirigentes, trabajadores de servicios, etc.) simplificando, de esta manera, la complejidad del espacio social.

Otra perspectiva de comprensión debe partir entonces de los puntos críticos siguientes :

- Ruptura con la posición de privilegiar los grupos reales (número, límites, miembros) en detrimento de las relaciones.

- Ruptura con la posición de concebir la clase teórica como una clase real, como un grupo movilizado.

- Ruptura con la reducción de comprensión del fenómeno de las clases solo al nivel de las relaciones de producción económica

- Ruptura con la ignorancia de las luchas simbólicas que existen en el espacio social y que tienen como objetivo las representaciones del mundo social y, por ende, la jerarquía de posiciones.

25 Marx, K. ; Engels, F., Obras escogidas en dos tomos, tomo 2, Ediciones en Lenguas Extranjeras, Moscú, 1955. 


\section{EL ESPACIO SOCIAL CUBANO Y EL SECTOR ECONÓMICO MIXTO O PRIVADO /}

Estos elementos los encontramos en la teoría de Pierre Bourdieu. Sin intentar exponer su sistema teórico por problemas de espacio dada su complejidad, sólo colocaremos algunos elementos de interés que puedan motivar reflexiones acerca de lo que está sucediendo en estos momentos en Cuba.

Una panorámica breve de esta teoría podría resumirse en que, para Bourdieu, las condiciones sociales de existencia son interiorizadas por los individuos bajo la forma de principios inconscientes de acción y de reflexión, como esquemas de la sensibilidad y del entendimiento, es decir, bajo forma de estructuras de la subjetividad, a las cuales brindó el nombre de habitus.

Pero el habitus, una vez estructurado a partir de las condiciones sociales de existencia, no va a cesar de producir representaciones, opiniones, creencias, gustos, deseos, en general, toda una subjetividad relativamente independiente del exterior, pero que no cesa de expresarse, de exteriorizarse en la acción de los individuos y grupos, y contribuye a producir y reproducir las estructuras sociales y las instituciones. Ellas, a su turno, impondrán sus condiciones de existencia objetivas, de manera que la realidad social se construye sin cesar a través de la acción de los individuos y de los grupos condicionados por la misma realidad social que les preexiste.

Las luchas por el poder sobre la legitimación de las representaciones del mundo social son un elemento esencial para la comprensión de esta dinámica, determinadas por la posesión de los diferentes capitales, y serán, para este autor, el objeto central de las luchas y del consenso.

Uno de los aspectos más importantes del trabajo de Pierre Bourdieu consiste en haber terminado con el encerramiento tradicional entre sociología y economía mostrando que el campo específicamente económico es susceptible del mismo análisis que los otros campos del mundo social, y que las estrategias propiamente económicas de apropiación de capital, son solamente un caso particular de estrategias a través de las cuales los diferentes agentes de los diferentes campos sociales se esfuerzan por adquirir o conservar las diferentes variedades de capital. Esto nos lleva 
a substituir de entrada la visión tradicional de las relaciones individuo - sociedad y nos aporta una visión nueva: aquella de las relaciones dialécticas entre un habitus socialmente estructurado y un campo históricamente constituido, es decir, nos lleva a buscar la relación entre lo social puesto en los cuerpos y lo social puesto en las cosas.

Si tratamos de ver la sociedad cubana bajo este prisma, se observa que las diferencias sociales en Cuba hasta 1989 no se dibujaban en el campo económico. Los logros de la Revolución elevaron el nivel de vida de la población y construyeron paulatinamente la creeencia en un sistema de valores diferentes al que impone ideológicamente el mercado. Sin embargo, si analizamos la sociedad cubana hasta esa fecha desde la perspectiva del campo político, podemos ver cómo las diferencias se dibujaban a partir de la detención capital político en volumen y estructura. No hay que discutir demasiado para comprender que quienes dirigían políticamente el país se sitúan en una relación diferente a los que resultan dirigidos, aunque para hacer justicia a la clase dirigente cubana debemos señalar su postura incorruptible, sacrificada y representante de los intereses de la mayoría de la población.

La entrada del mercado al país, si bien controlada estatalmente a partir del Período Especial, trajo como consecuencia la ya señalada potenciación de lo económico frente a lo político, sobre todo desde una perspectiva simbólica, lo que no quiere decir que una cosa vaya en detrimento de la otra. A pesar de la garantía de no acumulación legal de capital económico en la actualidad, las personas que pertenecen al grupo que analizamos en cuestión, principalmente los dirigentes empresarios, son detentores de formas simbólicas de capital económico asociados al mercado a pesar de no ser propietarios de ellas. Los grandes autos, los teléfonos, celulares, internet, el " saber hacer mercantil ", en fin, toda una simbólica cultural de la " modernidad capitalista" comienza a asociarse a este grupo social.

Aún el tiempo es breve y los mecanismos son fuertes, el alto grado de politización a favor de la Revolución de los dirigentes empresarios, el control estatal y la lucha contra la corrupción también son muy importantes, pero cabe preguntarse si estas simbólicas ideológicas podrán hacer que este grupo social empiece a actuar culturalmente en otro sentido, 


\section{EL ESPACIO SOCIAL CUBANO Y EL SECTOR ECONÓMICO MIXTO O PRIVADO /}

empiece a ver como " natural" las diferencias que se van dibujando con respecto a otros grupos, empiece a considerarse como grupo o como clase para sí.

Hasta el momento todo parece indicar que no, sin embargo, las circunstancias históricas que vive el país son muy complejas y su evolución depende en parte, evidentemente, de la evolución mundial, sobre todo frente a la globalización neoliberal. Los valores creados y asentados por la Revolución cubana son importantes y tienen 40 años de construcción conciente, la confianza en el Partido, el antimperialismo, la dirección histórica y la solidaridad internacionalista son simbólicas compartidas por la mayoría de los cubanos y asentadas con mucha fuerza, por lo que todo parece indicar que menos que estarse construyendo un nuevo grupo o clase social, es la propia clase dirigente quien comienza a extender sus fronteras ante la entrada de la inversión extranjera, en el entendido de mantenimiento del poder revolucionario y de las adquisiciones socialistas. El desafío radica entonces, a nuestro juicio, en garantizar que la apropiación simbólica " mercantil" por parte de los dirigentes empresarios del sector mixto o privado no lacere el sistema de valores socialistas y no provoque una fisura en la manera de pensar, de concebir el mundo y, en consecuencia, de actuar de este grupo.

\section{Las representaciones sociales del cambio. ¿Diferencias sociales o igualitarismo?}

A pesar de lo expuesto anteriormente, la percepción de los cubanos que están fuera del sector mixto o privado no se ajusta exactamente con lo que está pasando en la realidad objetiva. Una encuesta realizada a principios de 2000 habla por sí sola ${ }^{26}$. Veamos sus principales resultados :

El acuerdo de los entrevistados con los postulados del discurso oficial del gobierno cubano es indiscutible. El $96 \%$ de los encuestados afirma que la introducción de este sector privado o mixto resultó una necesidad para el país y consideran sus principales ventajas en el sentido de la obtención de divisas (50\%), la posibilidad de desarrollo para el país

26 La encuesta fue realizada a una muestra representativa de mayores de 16 años en Ciudad de La Habana y en el mismo propósito de la tesis doctoral anteriormente mencionada. 
(34\%) y la adquisición de conocimientos y tecnologías necesarias (7\%). Las desventajas señaladas coinciden también con lo reconocido por el discurso oficial: aparición de desigualdades sociales (49\%) y posibilidad de mercantilización de los valores ético morales (10\%). Sin embargo, el casi $30 \%$ de los entrevistados expresa que no hay ninguna desventaja con la introducción del sector en el país.

La mayoría de los encuestados (88\%) expresa que las ventajas de trabajar en el sector son estrictamente económicas, en tanto que el $51 \%$ de ellos dice que trabajar en el sector no tiene desventajas.

Los trabajadores del sector resultan, no obstante, evaluados por los encuestados con mucho más rigor. El $90 \%$ de la muestra considera que reciben más que el resto de la población, sólo un $45 \%$ dice que son sacrificados en su trabajo, únicamente un $24 \%$ considera que trabajan más, un $64 \%$ afirma no saber o dice categóricamente que no son ejemplo en la sociedad y sólo el $50 \%$ dice que los trabajadores de este sector son tan revolucionarios como el resto del pueblo cubano.

Las opiniones con respecto a si los que trabajan en el sector mixto o privado son iguales al resto de la población resultan divididas: el 51\% de los encuestados dice que sí en tanto que el $46 \%$ dice que no y un $2 \%$ no sabe. Quienes afirman que los del sector son diferentes basan mayoritariamente los argumentos explicativos en la variable material.

El 38\% de los encuestados piensa que el tipo de personas que entra al sector lo hace por su capital cultural y educacional, es decir, por sus condiciones intelectuales y laborales, en tanto que el $48 \%$ considera que el tipo de personas que entra son aquellos que tienen amigos o familiares en el sector, o son los propios dirigentes del país o sus familiares $\mathrm{o}$ amigos.

La mayor parte de los encuestados expresa que los mecanismos de entrada al sector no se cumplen (44\%) en tanto que sólo un 7\% de los encuestados dice que sí. Los restantes entrevistados no responden o afirman desconocer los mecanismos establecidos para entrar al sector mixto o privado. Solamente el $19 \%$ de los encuestados afirma que la entrada al 


\section{EL ESPACIO SOCIAL CUBANO Y EL SECTOR ECONÓMICO MIXTO O PRIVADO /}

sector se realiza por vías legales.

Cuando se les solicita a los encuestados cuál es el "deber ser" de un trabajador de este sector, la característica situada en primer lugar (32\%) es la política (ser revolucionario, estar acorde con los principios de la Revolución, etc.). En segundo lugar de importancia los entrevistados sugieren las características ético - morales (31\%) tales como: honestos, honrados, etc. El plano educacional pasa a un tercer lugar del deber ser según lo afirma el $28 \%$ de la muestra.

Sin embargo, cuando le preguntamos a los encuestados si consideran que los que trabajan actualmente en el sector mixto o privado tienen esas características, las respuestas son:

Se aprecia en la tabla anterior nuevamente la división de la opinión. Pero a pesar de la evaluación cuasi negativa realizada, el $40 \%$ de los encuestados

\begin{tabular}{lr}
\hline & $\%$ \\
Todos & 3 \\
La mayoría & 48 \\
La minoría & 33 \\
Ninguno & 2 \\
\hline
\end{tabular}
afirma desear trabajar en el sector mixto o privado, coincidiendo en gran parte los que desean esto con los que emiten valoraciones negativas respecto al sector.

Finalmente, el $36 \%$ de los encuestados expresa que todos debemos ser iguales, el $48 \%$ dice que debemos serlo en algunas cosas y en otras no y el $12 \%$ considera que no debemos ser iguales. Pero nótese lo que sucede cuando se le pregunta a la muestra cómo evalúa las diferencias sociales en Cuba:

Hasta aquí los resultados descriptivos de la encuesta. Veremos entonces a continuación un análisis más a fondo, realizado a

\begin{tabular}{|ll|}
\hline & $\%$ \\
Son muy grandes & 34 \\
Son normales, las que deberían existir & 39 \\
Son pocas & 20 \\
No hay diferencias sociales & 7 \\
\hline
\end{tabular}
través de métodos cualitativos factoriales:

Las representaciones sociales acerca de los cambios estructurales en Cuba 
y sus agentes están divididas. Existen dos grupos claramente definidos y con porcentajes de ocurrencia similares: el grupo que juzga negativamente el sector (41\%) y el que lo juzga positivamente (49\%). Veamos las características que los conforman:

Representaciones sociales respecto al sector mixto o privado

\begin{tabular}{|l|c|c|}
\hline & $\begin{array}{c}\text { Visión positiva } \\
\mathbf{4 9 \%}\end{array}$ & $\begin{array}{c}\text { Visión negativa } \\
\mathbf{4 1 \%}\end{array}$ \\
\hline ¿Fue necesario introducir el sector? & Sí & Sí \\
\hline Ventajas de su introducción & Económicas & Económicas \\
\hline Desventajas de su introducción & - & $\begin{array}{c}\text { Limita los derechos } \\
\text { de los cubanos }\end{array}$ \\
\hline Ventajas de trabajar en el sector & - & Económicas \\
\hline Desventajas de trabajar en el sector & No hay & Inseguridad laboral \\
\hline ¿Quiénes trabajan en él reciben más? & - & Sí \\
\hline ¿Quiénes trabajan en él son sacrificados? & Sí & No \\
\hline ¿Quiénes trabajan en él trabajan más? & Sí & No \\
\hline ¿Quiénes trabajan en él son ejemplo? & Sí & Sí \\
\hline $\begin{array}{l}\text { Quiénes trabajan en él son tan } \\
\text { revolucionarios como el resto del pueblo? }\end{array}$ & Sí & Materialmente \\
\hline ¿Quiénes trabajan en él son diferentes? & No & Los que tienen amigos \\
\hline ¿En qué son diferentes? & En nada & \\
\hline $\begin{array}{l}\text { Tipo de personas que } \\
\text { trabaja en el sector }\end{array}$ & $\begin{array}{c}\text { Los que tienen mejores } \\
\text { condiciones educacionales }\end{array}$ & La minoría \\
\hline $\begin{array}{l}\text { ¿Los que trabajan en el sector tienen las } \\
\text { características que Ud. considera ideales } \\
\text { para integrar el sector? }\end{array}$ & & Sí \\
\hline ¿Le gustaría trabajar en el sector? & La mayoría & - \\
\hline $\begin{array}{l}\text { ¿Se cumplen los mecanismos } \\
\text { de entrada al sector? }\end{array}$ & No sé, porque no \\
\hline $\begin{array}{l}\text { Las diferencias sociales en Cuba son: } \\
\text { los conozco }\end{array}$ & $\begin{array}{c}\text { Son normales, las que } \\
\text { tendrían que existir }\end{array}$ & Son muy grandes \\
\hline Edad & Jubilados & De 31 a 50 años \\
\hline Ocupación & & Trabajador \\
\hline
\end{tabular}

\section{¿Una clase como representación social ?}

¿Por qué si los dirigentes empresarios no tienen objetivamente la posibilidad de acumulación de capital económico por vías diferentes de la corrupción la mitad de las representaciones sociales considera que sí?

¿Por qué si las diferencias sociales en Cuba son moderadas y muy lejos de las existentes en los países del área hay más de un tercio de los encuestados que las ve muy grandes? 


\section{EL ESPACIO SOCIAL CUBANO Y EL SECTOR ECONÓMICO MIXTO O PRIVADO I}

¿Por qué si la mayor parte de los dirigentes empresarios son revolucionarios, militantes y personas designadas para ocupar esta función vital para el desarrollo del país, la mitad de las representaciones sociales los considera poco sacrificados, que no son ejemplo, que no trabajan más que los demás y que no son tan revolucionarios como el resto del pueblo cubano?

¿Por qué son considerados diferentes por una parte del imaginario colectivo?

Entre otras, estas interrogantes surgen del contraste de las posibilidades objetivas del grupo de agentes del cambio (dirigentes empresarios), y de lo expresado a nivel de las representaciones sociales.

Una primera hipótesis, nos llevó a suponer estas actitudes como consecuencia de representaciones impuestas a través de los medios de comunicación. Sin embargo, un análisis exhaustivo de la prensa plana cubana de todo 1999 y parte del 2000 falsifica rápidamente esto.

Los medios de comunicación cubanos tienen un noble tratamiento hacia este tema. Los artículos y alusiones respecto al sector son generalmente informativos y no encontramos prácticamente ninguno que exprese opinión. Cuando este es el caso, la opinión se orienta hacia los logros del sector y sus cuadros, por lo que resulta fácil concluir que la conformación de estas representaciones negativas no se ha realizado por esta vía.

Una segunda hipótesis estuvo orientada hacia el discurso politico. Es sabido que el tema de las diferencias sociales ha sido tratado en innumerables ocasiones por los dirigentes de la Revolución, en el entendido de explicar el cambio en la política hasta entonces extremadamente igualitarista. No obstante a ello, apreciamos concordancia entre los postulados del discurso y las representaciones sociales, ya que tanto quienes evalúan positiva como negativamente el sector, coinciden en la comprensión de la necesidad de introducirlo para salvar el proceso cubano así como que su entrada sería generadora de diferencias sociales.

¿De dónde viene entonces este sentimiento negativo de evaluación del sector, cuya coherencia expresa extremos como los que hemos expuesto? 
Una primera pista de reflexión podría ser toda la simbólica que entorna a este grupo social y a su macro estructura. No podemos decir que los cubanos han vivido ajenos a las diferencias y que el proceso igualitarista fue absoluto. La combinación del estímulo moral con el material fue una constante, con altas y bajas, del proceso revolucionario hasta los años 90 y los sectores que más aportaban al desarrollo social tenían mayores posibilidades materiales que los restantes. Por ejemplo, un trabajador destacado del azúcar (renglón principal de la economía cubana hasta los anos 90) recibía un mayor salario que los demás y tenía mayor estimulación material. Y las representaciones sociales no cuestionaban esta política ni cuestionaban a estos individuos.

Sin embargo, en estos momentos la apertura de este sector se realiza principalmente vinculada al desarrollo acelerado del turismo internacional en Cuba, pasando esta industria sin humo a convertirse en el renglón más importante de aportes financieros dentro de la economía de la isla. Y el turismo en Cuba no está considerado en estos momentos como fuente de placer para la mayoría de la población sino como fuente de ingresos para la subsistencia y el desarrollo del país, aunque debemos señalar con justeza que el fenómeno de considerar "turistas" a los trabajadores del turismo y a sus empresarios no sólo es propio al caso cubano.

La simbólica que se muestra en este caso es la del empresario exitoso y no la del trabajador sacrificado del azúcar. El empresario trabaja en aire acondicionado, el azucarero suda bajo el sol; el empresario debe reunirse a cenar con clientes extranjeros, el azucarero come dentro del campo de caña; el empresario regresa a su casa en auto, el azucarero a pie o en el transporte colectivo, .... En fin, el azucarero es la imagen del sacrificio cotidiano en la Revolución, el empresario es la imagen del mercado, es la imagen del éxito y es también la imagen contraria a las importantes carencias que sufre el pueblo cubano en la actualidad.

Pero lo que no se puede perder de vista es que, salvo a través de la corrupción, el empresario es sólo esa imagen simbólica, que esconde sacrificio, jornadas laborales extensas, tensión por la cantidad de controles, tensión por la responsabilidad ante el país y su dirección... 


\section{EL ESPACIO SOCIAL CUBANO Y EL SECTOR ECONÓMICO MIXTO O PRIVADO /}

No resultará fácil en el proceso cubano borrar las huellas de 30 años de igualitarismo y paternalismo estatal. Las diferencias necesarias que se abren en el espacio social son difíciles de asimilar por la población, más aún en las condiciones materiales de vida en que se insertan la mayoría de los cubanos. Las más pequeñas distancias son consideradas enormes y el imaginario colectivo comienza a construir representaciones que no necesariamente se corresponden con la realidad social y que van conformando una imagen, un grupo, una clase construida...

Es por ello que cabe preguntarse entonces la siguiente interrogante teórica: ¿Hasta dónde una clase social es una construcción solamente objetiva, es decir, un grupo con límites y fronteras?, ¿Cuál es la línea que divide la clase social como grupo objetivo de la clase social construida en el imaginario colectivo?, ¿Si el imaginario social cree que la clase existe, eso basta para afirmar su existencia como grupo?

De las interrogantes podemos derivar reflexiones interesantes, pero para los intereses del socialismo cubano hay que continuar y reafirmar la política actual. La forma de concepción de la propiedad y la lucha contra la corrupción y el enriquecimiento ilícito fundamentan las bases de negación de la acumulación capitalista. Habrá que demostrar a la población cubana que los dirigentes empresarios representan sus intereses desde la perspectiva socialista y que sólo existen como instrumentos de poder garantes del desarrollo social. Que lo sean y que se perciba así. He ahí el desafío. 